\title{
ANÁLISIS MINERALÓGICO CUALITATIVO Y CUANTITATIVO EN ARCILLAS POR DIFRACCIÓN DE RAYOS X Y EL MÉTODO RIETVELD USANDO EL PROGRAMA FULLPROF
}

\author{
Rubén Quille R ${ }^{\mathrm{a}}$, Angel Bustamante $\mathrm{D}^{\mathrm{a}}$, \\ ${ }^{a}$ Laboratorio de Arqueometría, Facultad de Ciencias Físicas, Universidad Nacional Mayor de San Marcos, \\ Apartado Postal 14-0149, Lima, Perú.
}

\begin{abstract}
Resumen
En el presente trabajo se realizó la identificación cualitativa y cuantitativa de las principales fases mineralógicas presentes en una muestra de arcilla, para mostrar un método que permitió ajustar su estructura cristalina y a la vez determinar las concentraciones de las fases mineralógicas presentes en la muestra de arcilla. Los datos se obtuvieron con un Difractómetro Universal y se aplicó el método Hanawalt para la identificación de las fases, para ello utilizamos los software DRXWIN 2.2 y el CreaFit 2.2. El refinamiento Rietveld se realizó usando el programa FullProf, el cual permitió refinar los parámetros de red, las posiciones atómicas y determinar las concentraciones de las fases mineralógicas presentes en la muestra, a la vez permite representar gráficamente la estructura cristalina refinada de cada fase mineralógica y el Mapa de Fourier.
\end{abstract}

\section{Palabras claves:}

\begin{abstract}
In the present work were making the qualitative and quantitative identification of the main mineralogical phases present in a specimen of clay, to show a method to adjust their crystalline structures and at the same time determine the concentrations of the mineralogical phases present in the specimen of clay. The data were obtained with an Universal Difractómeter and the Hanawalt method was applied for the identification of the phases, for it we use the softwares DRXWIN 2.2 and CreaFit 2.2. The Rietveld refinement were making using the FullProf program, it allowed refine the net parameters, atomic positions and it determine concentrations of the mineralogical phases present in the specimen, at the same time it allows represent graphically refine crystalline structure of each mineralogical phase and the Fourier Map.
\end{abstract}

Keywords

\section{Introducción}

En Ayacucho la cerámica es la principal actividad del pueblo. El barro de colores rojos y crema le da una característica especial a estas piezas, que a pesar de sus formas simples y casi infantiles, poseen una gran fuerza expresiva. Ayacucho tiene como capital la ciudad de
Ayacucho, está localizada a 500km de Lima y a 2,761 m de altitud. El distrito de Quinua se encuentra a $37 \mathrm{~km}$ de la ciudad de Ayacucho y a $3,300 \mathrm{~m}$ de altitud, en ella se encuentran los talleres de cerámica de los artesanos (conocido también como pueblo de alfareros) y la mayoría de las canteras. A la vez Ayacucho cuenta con el Centro Experimental de Cerámica que pertenece a la Facultad de Ingeniería Química y

E-mail: rubenquille@hotmail.com 
Metalúrgica de la Universidad Nacional San Cristóbal de Huamanga (UNSCH). El Centro Experimental de Cerámica de la Universidad Nacional de San Cristóbal de Huamanga, inicio sus actividades el 30 de enero de 1961, gracias a un acuerdo entre el Real Gobierno de Dinamarca y las Naciones Unidas contando con el respaldo del Gobierno Peruano. Es un centro de enseñanza, investigación, proyección social, y desarrolla un pequeño programa de producción. En la producción de objetos cerámicos el Centro Experimental de Cerámica, utiliza arcillas rojas y arcillas blancas. La arcilla es una materia prima básica para la industria cerámica, que se presenta en la naturaleza, todas se parecen en que contienen silicato de aluminio acuoso como principal componente (ref.1). En este trabajo se incluye los resultados obtenidos del análisis cualitativo y cuantitativo de una muestra de arcilla formulada en el Centro Experimental de Cerámica, la materia prima fue obtenida de las canteras del Distrito de Quinua. La difracción de polvo de rayos $\mathrm{X}$ es el procedimiento más útil para obtener información cualitativa y cuantitativa en mezclas multifásicas (ref. 2). Sin embargo, en arcillas, la determinación de la composición del material es un problema enormemente complejo debido al fuerte solapamiento entre las reflexiones Bragg de las diferentes fases. El uso del método de Rietveld nos permite resolver este problema (ref. 3 y 4). Entre las ventajas que ofrece dicho método destacaremos: El ajuste del espectro de difracción completo minimiza el problema del solapamiento entre picos de difracción. Efectivamente, todas las reflexiones correspondientes a las diferentes fases se incluyen explícitamente en el refinamiento, reduciendo así la incertidumbre en los valores de las fracciones en peso. Los objetivos de este trabajo es realizar el análisis cualitativo y cuantitativo de una muestra de arcilla mediante difracción de rayos X y aplicando el método de Rietveld.

\section{Parte Experimental}

Para el análisis instrumental de la muestra se procedió de la siguiente manera:

\section{a. Preparación de la muestra:}

La muestra se sometió al proceso de cuarteo. Para el análisis por difracción de rayos X (DRX) la muestra fue pulverizada en un mortero de ágata y pasado por un tamiz de $45 \mathrm{~mm}$.

\section{b. Análisis por DRX:}

Para las mediciones por DRX, se utilizó un difractómetro convencional "Rigaku" de la Facultad de Ciencias Físicas de la Universidad Nacional Mayor de San Marcos, con geometría $(\theta-2 \theta)$. El difractograma fue tomado con radiación $\mathrm{K}_{\alpha}$ de $\mathrm{Cu}$, con una resolución de $0,04 \%$ paso $4 \mathrm{~s}$, y cubre un alcance de $5^{\circ}<2 \theta<$ $65^{\circ}$.

\section{c. Análisis Mineralógico Cualitativo:}

Para el análisis de cualitativo aplicamos el método de Hanawalt, (ref. 5) el cual se procede de la siguiente manera: Se inicia el proceso trabajando con los picos que pertenecen a la fase que supuestamente esta presente en el difractograma, para ello utilizamos los software ORIGIN 6.0, DRXWIN 2.2 y CREAFIT 2.2. Haciendo una selección adecuada de los picos más intensos utilizamos el CREAFIT donde se ha colocado una base de datos mineralógicos obtenidos de otra base de datos más amplio denominado PDF2, (ref. 6) luego elegimos el patrón mineralógico que más se aproxime a dichas intensidades. Para las siguientes fases se continúo con el mismo proceso. Para poder visualizar el difractograma en el DRXWIN 2.2 se utilizó el programa "POWDER 4", el objetivo era cambiar el formato de datos emitidos por el difractómetro al formato con extensión "*.udf" de esta manera poder visualizar el difractograma.

\section{d) Análisis Mineralógico Cuantitativo aplicando el Método Rietveld usando el Programa FullProf:}

Los datos de difracción de rayos $\mathrm{X}$ de polvos de la muestra de arcilla fue refinado por el Método Rietveld usando el programa FullProf (ref. 7). Este programa de refinamiento de estructuras cristalinas basado en el método de ajuste por mínimos cuadrados para optimizar la información proporcionada en los patrones de difracción. Sin embargo, estos valores no tendrían validez si no fuesen generados por 
valores coherentes desde el punto de vista físico y estructural. En este sentido debe tenerse buen criterio al variar los múltiples parámetros que refina el programa. Teniendo en cuenta este aspecto se hizo un acompañamiento cuidadoso tanto del perfil de difracción simulado como del refinamiento de los valores. Los pasos a seguir en el refinamiento de los parámetros cristalinos fueron los siguientes:

- Se escogió como función de perfil la función pseudo- Voigt.

- Background, se modelizó mediante una función polinómica de cuarto grado.

- Se refinó los factores de escala de cada una de las fases.

- Parámetros instrumentales globales (desplazamiento de la posición de cero del difractómetro).

- Parámetros de red para todas las fases.

- Parámetros de perfil (parámetros de anchura de Caglioti).

- Las posiciones atómicas.

- Al finalizar se soltó todos los parámetros a refinar y se simulo hasta llegar a la convergencia.

El método de Rietveld da información acerca de las proporciones relativas de las fases, se obtiene a partir de los factores de escala determinados en el último ciclo del ajuste, empleando la siguiente expresión (ref. 8):

$$
X_{1}=\frac{s_{1} Z_{1} M_{1} V_{1}}{\sum s_{j} Z_{j} M_{j} V_{j}}
$$

donde $Z_{1}, M_{1}$, y $V_{1}$ son, para la fase 1 , el número de moléculas en la celda unitaria, el peso molecular y el volumen de la celda unitaria, respectivamente, siendo $\mathrm{s}_{1}$ el factor de escala. $\mathrm{La}$ sumatoria en $\mathrm{j}$ se extiende a todas las fases incluidas en el modelo. Por otra parte, el grado de concordancia entre los datos experimentales y el modelo empleado en el método de Rietveld se midió empleando el índice siguiente:

Factor de Bragg

$$
R_{B}=100 \frac{\sum_{h}\left|I_{o b s, h} \cdot-I_{c a l, h}\right|}{\sum\left|I_{o b s, h}\right|}
$$

donde: ' $I_{o b s, h}$ ' es la intensidad integrada observada es un factor calculado de la formula Rietveld:

$$
I_{o b s, h^{\prime}}=I_{c a l, h} \sum_{i}\left\{\frac{\Omega\left(T_{i}-T_{h}\right)\left(y_{i}-b_{i}\right)}{\left(y_{c, i}-b_{i}\right)}\right\}
$$

donde:

- $I_{c a l, h}$ intensidad integrada calculada

- $y_{i}$ intensidad observada

- $b_{i}$ es la intensidad de background

- $y_{c, i}$ intensidad calculada

- $\Omega$ es la función perfil de reflexión

- $T_{i}$ es el ángulo $2 \theta$ observado

- $T_{h}$ es el ángulo $2 \theta$ calculado

A parte de este factor debemos de tener en cuenta la curva de diferencia entre el espectro experimental y el espectro calculado, dicha diferencia debe ser lo más mínimo posible.

\section{Resultados y Discusión}

Una cuidadosa inspección en el difractograma (figura 1), vemos que la muestra de arcilla, contiene cuarzo (oxido de silicio, $\mathrm{SiO}_{2}$ ) como fase principal. Como segunda fase hemos obtenido Albita $\left(\mathrm{NaAlSi}_{3} \mathrm{O}_{8}\right)$ y las otras fases encontradas fueron: Caolinita $\left(\mathrm{Al}_{2} \mathrm{Si}_{2} \mathrm{O}_{5}(\mathrm{OH})_{4}\right)$, Muscovita $\quad\left(\begin{array}{llllll}\mathrm{KAl}_{2} & \mathrm{Si}_{3} & \mathrm{Al} & \mathrm{O}_{10} & \left.(\mathrm{OH})_{2}\right) & \text { y }\end{array}\right.$ Montmorillonita $\left(\mathrm{MgOAl}_{2} \mathrm{O}_{3} 5 \mathrm{SiO}_{2} \mathrm{H}_{2} \mathrm{O}\right)$. En la figura 2 se visualiza la indexación de las fases encontradas. Estos difractogramas fueron normalizados a 100 unidades arbitrarias (u.a.). En la Fig. 3 se muestra los resultados del refinamiento Rietveld, vemos que la curva de diferencia (color azul) obtenida entre la intensidad observada y la intensidad calculada por el programa FullProf es mínima, todos los picos encajan. En este análisis se consideró tres fases mineralógicas, en la tabla 1 se presentan las fracciones en peso de las fases mineralógicas refinadas obtenidas a partir de los factores de escala. Por otra parte, vemos que el índice de Factor de Bragg indica aproximadamente nueve para las tres fases refinadas, este valor es razonable para un buen refinamiento. En la tabla 2 se indica los parámetros de red de cada fase refinada. En las figuras 4, 5 y 6 se muestran las estructuras cristalinas que fueron diseñadas por el programa FullProf en donde utiliza los valores de los parámetros red y las posiciones atómicas después de refinarlas. En la Figura 7 se muestra el mapa de Fourier en 3 dimensiones (3D), 
correspondiente a las tres fases mineralógicas refinadas, obtenidos por el programa FullProf.

\section{Conclusiones}

La identificación de muestras monofásicas puede ser realizada sin mucha dificultad con la búsqueda en la base de datos mineralógicos del software PDFWIN aplicando el método Hanawalt. Las dificultades surgen cuando se trata de muestras multifásicas, en cuyo caso los métodos computacionales son sumamente útiles, como fue mostrado en este trabajo. En el presente trabajo se muestra que la aplicación del método de Rietveld nos permite obtener las fracciones en peso de las diferentes fases presentes en una muestra de Arcilla. La difracción de Rayos $X$ es una técnica de caracterización muy útil, que nos proporciona una información estructural muy detallada las estructuras cristalinas. El Programa FullProf nos da automáticamente después de haber refinado las fases mineralógicas, el grafico de la estructura cristalina de cada fase mineralógica y el mapa de Fourier en 3D, esto nos permite visualizar la forma de estructura cristalina y la forma de la densidad electrónica.

\section{AGRADECIMIENTOS}

Los autores desean expresar su agradecimiento al Consejo Nacional de Ciencia y Tecnología por la financiación recibida a través del proyecto: "Mejora en la calidad de los productos cerámicos para la exportación de los artesanos de Ayacucho, Arequipa y Huancavelica usando técnicas físicas y químicas. I Etapa", sin ello quizás no hubiera sido posible la elaboración de este trabajo.

\section{Referencias}

[1] Hald, P. "Técnica de la Cerámica", publicado por Ediciones Omega, Barcelona, 1977.

[2] Klug, H.P., y L.E. Alexander "X-ray diffraction procedures for polycrystalline and amorphous materials" (Chapter 7). John Wlley and Sons, New York (1974).

[3] Rietveld, M. "A profile refinement method for nuclear and magnetic structures". J. Appl. Cryst. 2 65-71 (1969).

[4] Bish, D.L. y S.A. Howard "Quantitative phase analysis using fue Rietveld method". J. Appl. Cryst. 21 86-91 (1988).
[5] Cullity B.D., "Elements of X - Ray Diffraction", Addison Wesley, Publishing Company, Inc. Massachusetts, 1967.

[6] Powder Diffraction File, Publicado por Joint Committee on Powder Diffraction Standar Philadelphia, 1974.

[7] Rodríguez, J. " An Introduction to the Program FULLPROF 2000" Laboratoire Léon Brillouin (CEA-CNRS) CEA/Saclay,91191 Gif sur Yvette Cedex, France (2001).

[8] Hill, R.J. "Expanded use of the Rietveld method in studies of phase abundance in multiphase mixtures". Powder Diffract. 6 [2] 74-77 (1991)

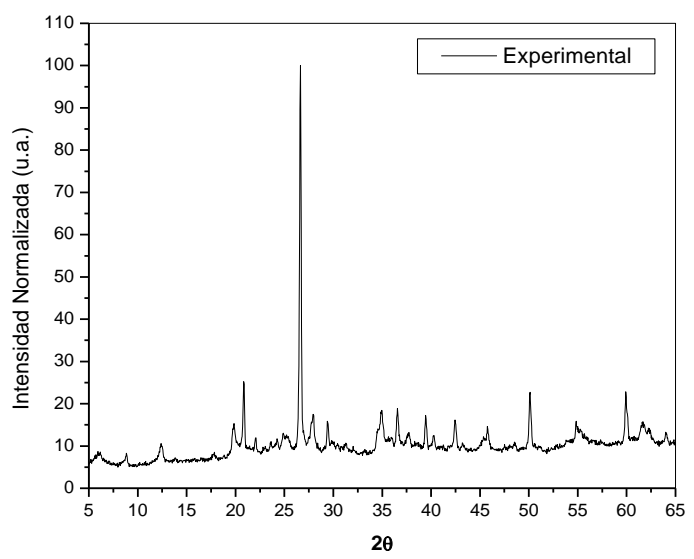

Fig.1. Difractograma correspondiente a la muestra de arcilla.

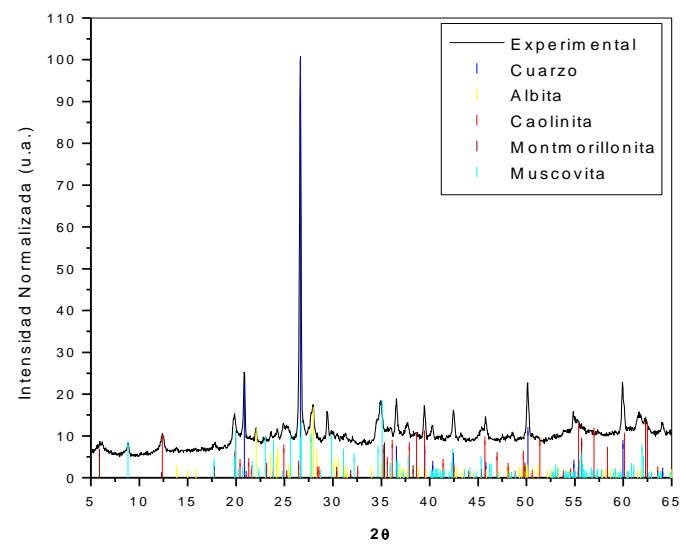

Fig. 2. Difractograma indexado con las fases mineralógicas identificadas. 
Tabla 1- Fracciones en peso de cada fase refinada y el factor de Bragg para cada fase, respectivamente.

\begin{tabular}{|l|l|}
\hline \multicolumn{1}{|c|}{ Modelo } & $(\%$ en peso $)$ \\
\hline Cuarzo & $15,88 \pm 0,02$ \\
\hline Albita Factor de Bragg $-\boldsymbol{R}_{\boldsymbol{B}}$ \\
\hline Caolinita & $76,63 \pm 0,08$ \\
\hline \multicolumn{2}{|c|}{9,11} \\
\hline Cuarzo & 9,69 \\
\hline Albita & 9,40 \\
\hline Caolinita & 9,20 \\
\hline
\end{tabular}

Tabla 2 - Parámetros de red de cada fase mineralógica refinada.

\begin{tabular}{|c|c|}
\hline Fase :Cuarzo & Parámetros de red \\
\hline \multicolumn{2}{|c|}{$\begin{array}{l}\mathrm{A}=4,91369 \mathrm{~A}, \mathrm{~B}=4,91369 \mathrm{~A}, \mathrm{C}=5,4069 \mathrm{~A} \\
\alpha=90,0000{ }^{\circ} \beta=90,0000{ }^{\circ} \gamma=120,0000\end{array}$} \\
\hline Fase :Albita & \begin{tabular}{l|l} 
Fase : Albita & Parámetros de red \\
\end{tabular} \\
\hline \multicolumn{2}{|c|}{$\begin{array}{l}\mathrm{A}=8,22603 \stackrel{\circ}{\mathrm{A}}, \mathrm{B}=13,19018 \mathrm{~A}, \\
\mathrm{C}=7,28865 \mathrm{~A} \\
\alpha=93,25482{ }^{\circ} \beta=118,47038{ }^{\circ} \gamma=90,1444 \circ\end{array}$} \\
\hline Fase :Caolinita & Parámetros de red \\
\hline $\begin{array}{l}A=5,05058 \mathrm{~A}, \mathrm{~B} \\
\alpha=92,25465\end{array}$ & $\begin{aligned} 93 \mathrm{~A}, \mathrm{C}=7,37974 \mathrm{~A} \\
30794 \quad \circ \gamma=88,33663\end{aligned}$ \\
\hline
\end{tabular}

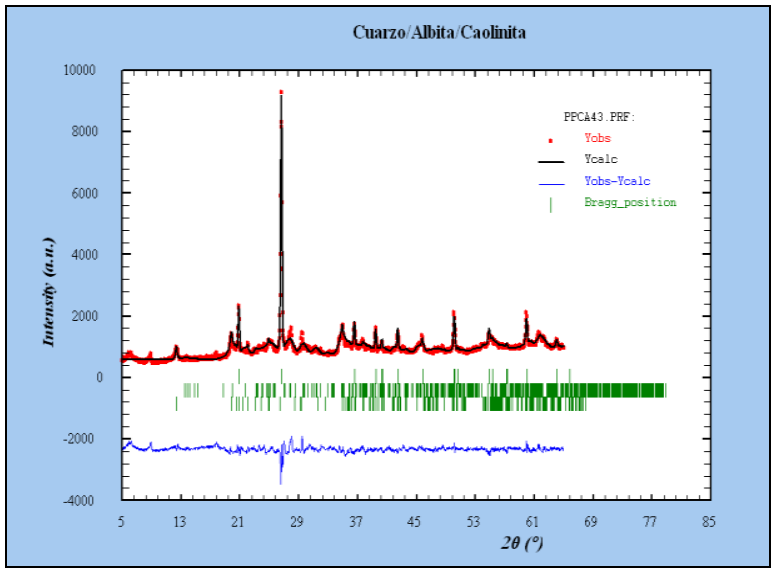

Fig. 3. Refinamiento Rietveld de la muestra de arcilla, por difracción de rayos X. La línea roja representa los datos experimentales, la línea negra el calculado, la diferencia abajo del espectro.

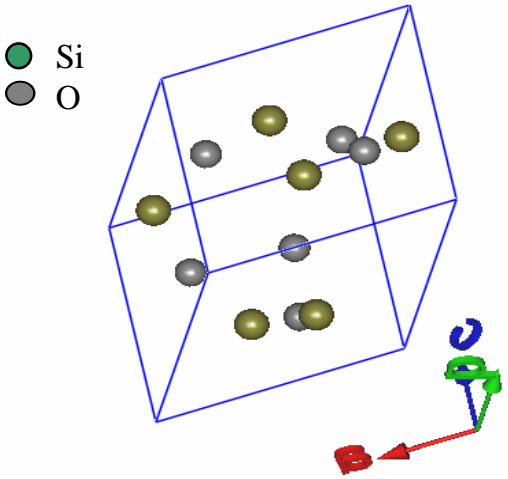

Fig. 4. Estructura cristalina del Cuarzo obtenida por el programa FullProf.

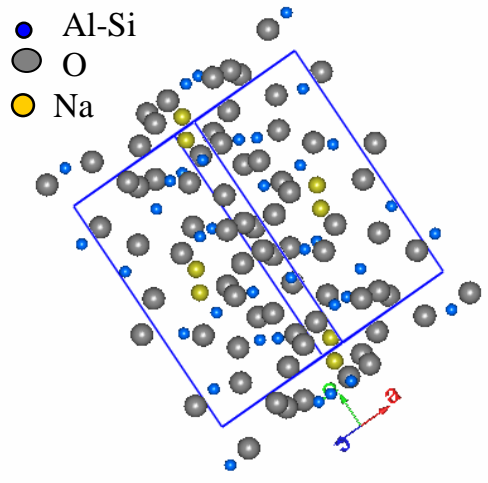

Fig. 5. Estructura cristalina de la Albita obtenida por el programa FullProf.

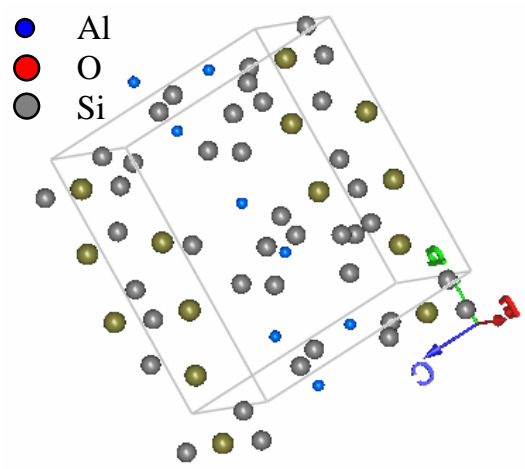

Fig. 6. Estructura cristalina de la Caolinita obtenida por el programa FullProf.

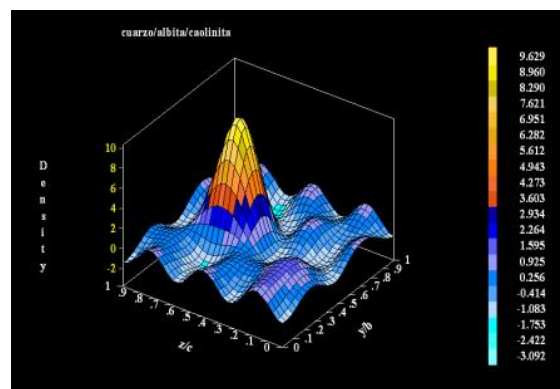

Figura 7. Mapa de Fourier en 3D correspondiente a las tres fases mineralógicas refinadas. 